\title{
Experience of patisiran with transthyretin stabilizers in patients with hereditary transthyretin-mediated amyloidosis
}

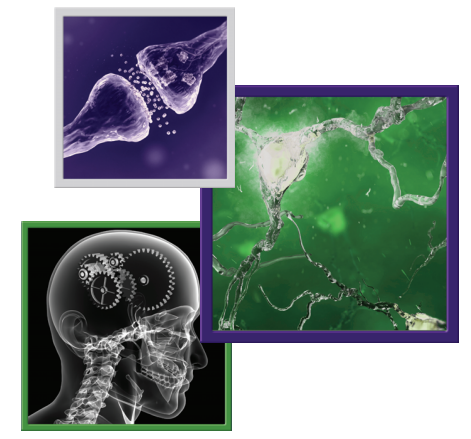

\author{
Hollis Lin*,1, Madeline Merkel ${ }^{1}$, Cecilia Hale ${ }^{1}$ \& Jing L Marantz ${ }^{1}$ \\ ${ }^{1}$ Alnylam Pharmaceuticals, Cambridge, MA 02142, USA \\ *Author for correspondence: Tel.: +1 617704 4853; holin@alnylam.com
}

\section{Practice points}

- Hereditary transthyretin-mediated (hATTR) amyloidosis is a rare, inherited and progressively debilitating disease.

- Patisiran has been shown to halt or reverse polyneuropathy and improve quality of life in the majority of patients in the Phase III randomized, controlled study (APOLLO); patisiran is approved in many countries globally for the treatment of patients with hATTR amyloidosis with polyneuropathy.

- Post hoc analyses from the Phase II open-label extension (OLE) study examined the use of patisiran alone or with a concomitant transthyretin (TTR) stabilizer (either tafamidis or diflunisal).

- Post hoc analyses from the Phase III APOLLO study evaluated the use of patisiran in patients with and without prior TTR stabilizer use (tafamidis or diflunisal).

- In the Phase II OLE, safety associated with patisiran use, alone or with a concomitant TTR stabilizer, was consistent with the safety profiles of each monotherapy as reported in their respective pivotal clinical studies.

- Average serum TTR reduction over 24 months was similar regardless of whether a patient received patisiran alone or with concomitant tafamidis or diflunisal in the Phase II OLE.

- In APOLLO, the safety and tolerability of patisiran were consistent regardless of prior tafamidis or diflunisal use.

- At 18 months in APOLLO, stabilization or improvement in polyneuropathy symptoms and quality of life relative to patients' baseline (as measured by mean values of modified Neuropathy Impairment Score +7 and Norfolk Quality of Life-Diabetic Neuropathy questionnaire) was observed on average with patisiran, regardless of prior TTR stabilizer use, whereas placebo-treated patients progressed on these measures across all subgroups on average.

- Delays in patisiran therapy have been associated with deterioration of neurological function and quality of life, which underscores the importance of timely initiation of patisiran for the treatment of this rapidly progressive disease.

- These data suggest that patients benefit from patisiran treatment regardless of concomitant or prior use of TTR stabilizers.

Aim: Examine safety and pharmacodynamics of patisiran alone or with concomitant transthyretin stabilizers from the Phase II open-label extension study and safety and efficacy of patisiran in patients with prior transthyretin stabilizer use from the Phase III APOLLO study. Patients \& methods: Post hoc analyses in patients with hereditary transthyretin-mediated amyloidosis with polyneuropathy. Results: Patisiran safety was consistent regardless of concomitant or prior transthyretin stabilizers. In the Phase II openlabel extension ( $n=27)$, transthyretin reduction was similar over 24 months, regardless of concomitant transthyretin stabilizers. In APOLLO $(n=225)$, patisiran-treated groups showed stabilization or improvements in neurological function (modified Neuropathy Impairment Score +7 ) and quality of life (Norfolk Quality of Life-Diabetic Neuropathy questionnaire) at 18 months, regardless of prior transthyretin stabilizers. Conclusion: Patients benefit from patisiran regardless of transthyretin stabilizer use.

First draft submitted: 17 April 2020; Accepted for publication: 11 May 2020; Published online: 10 June 2020

Keywords: amyloidosis • concomitant therapy • diflunisal • hereditary • monotherapy • patisiran • tafamidis • transthyretin • transthyretin stabilizer 
Hereditary transthyretin-mediated amyloidosis \& the treatment landscape Hereditary transthyretin-mediated (hATTR) amyloidosis, also known as ATTRv (v for variant) amyloidosis, is a rare, inherited and progressively debilitating disease. It occurs when mutations in the transthyretin (TTR) gene cause misfolded TTR to accumulate as amyloid deposits and damage multiple organs and tissue types (e.g., nerves and heart) [1,2]. hATTR amyloidosis has significant morbidity and mortality and affects approximately 50,000 people worldwide [1,2]. The median survival is 4.7 years following diagnosis, which is reduced to 3.4 years in patients with cardiomyopathy [3-6]. As a result of its multisystem and multi-organ involvement, the disease often has a heterogeneous clinical presentation [2], with the majority of patients developing a mixed phenotype of both polyneuropathy and cardiomyopathy [7-10].

Current treatment strategies for hATTR amyloidosis include pharmacotherapies directed at the disease pathophysiology. TTR stabilizers work by stabilizing the TTR protein complex and thereby preventing dissociation of the TTR tetramer into amyloidogenic monomers. Multiple studies have shown that patients treated with these therapies experience slowing, but not halting, of neuropathy progression on average [11-13]. The TTR stabilizer tafamidis is approved in select countries outside the USA for the treatment of early-stage polyneuropathy in patients with hATTR amyloidosis [13-17]. It has also been approved in the USA, Europe and Japan for the treatment of cardiomyopathy of transthyretin-mediated (ATTR) amyloidosis based on Phase III study results demonstrating reductions in all-cause mortality and cardiovascular-related hospitalizations [15,17-19]. Diflunisal is a nonsteroidal anti-inflammatory drug with TTR-stabilizing properties that is used off-label to treat ATTR amyloidosis [2]. It was shown to slow neurological progression relative to placebo in one randomized, controlled study of patients with hATTR amyloidosis with polyneuropathy [11]. TTR silencing therapies, which work to block production of diseasecausing mutant and wild-type (wt) TTR proteins through distinct mechanisms, have been approved in a number of countries for the treatment of hATTR amyloidosis with polyneuropathy [20-27]. Inotersen, an antisense oligonucleotide that reduces circulating TTR levels, significantly slowed neurological disease progression and worsening of quality of life (QOL) compared with placebo in the pivotal Phase III NEURO-TTR trial; however, the majority of patients treated with inotersen experienced progression of polyneuropathy over the course of the study [8]. Patisiran is a double-stranded small-interfering RNA that reduces serum TTR levels by inhibiting hepatic synthesis of the disease-causing mutant and wt TTR proteins through RNA interference [28-30]. The Phase III APOLLO study showed that patisiran was able to halt or reverse polyneuropathy and improve QOL from baseline in the majority of patients [7]. Additionally, in a prespecified cardiac subpopulation in APOLLO, patisiran treatment resulted in improvements in several measures of cardiac structure and function compared with placebo [31].

The introduction of new therapies with different mechanisms of action has increased interest to understand the potential position of each therapy within the therapeutic landscape to optimize patient care. Here we present post hoc analyses evaluating the safety and pharmacodynamics (PD) of patisiran alone or with concomitant TTR stabilizers from the Phase II open-label extension (OLE) study, and the safety and efficacy of patisiran in patients with prior TTR stabilizer use from the Phase III APOLLO study.

\section{Patients \& methods}

Phase II OLE study design

The Phase II OLE (ClinicalTrials.gov: NCT01961921) was a 24-month, multicenter, international extension of the Phase II study (NCT01617967) of patisiran $0.3 \mathrm{mg} / \mathrm{kg}$, administered intravenously (IV) every 3 weeks (q3W), in patients with hATTR amyloidosis with polyneuropathy [32]. Patients were included in the prespecified cardiac subpopulation if they had pre-existing cardiac amyloid involvement, defined as baseline left ventricular wall thickness $\geq 13 \mathrm{~mm}$, normotensive or well-controlled hypertension, and, per investigator determination, no medical history of aortic valve disease. The primary objective of the Phase II OLE was to evaluate the safety of long-term dosing with patisiran. Assessment of PD effect, such as serum TTR reduction, was a secondary objective of the study. Patients were permitted to receive concomitant tafamidis or diflunisal during the Phase II OLE study if the patient had started the treatment prior to study entry.

APOLLO Phase III study design

APOLLO (ClinicalTrials.gov: NCT01960348) was a Phase III, randomized, placebo-controlled study of patisiran $0.3 \mathrm{mg} / \mathrm{kg}$ IV q3W (2:1 randomization to patisiran or placebo) in patients with hATTR amyloidosis with 
polyneuropathy [7]. Patients were included in the prespecified cardiac subpopulation if they had evidence of cardiac amyloid involvement, which was defined as left ventricular wall thickness $\geq 13 \mathrm{~mm}$ and no aortic valve disease or hypertension in their medical history. The primary endpoint of APOLLO was change in modified Neuropathy Impairment Score +7 (mNIS+7) from baseline at 18 months. mNIS +7 is a composite measure of polyneuropathy that assesses motor, sensory and autonomic neuropathy, and has been specifically designed for use in patients with hATTR amyloidosis with polyneuropathy; a higher score indicates worsening impairment (range: 0 to 304) [33]. A key secondary endpoint was change in the Norfolk Quality of Life-Diabetic Neuropathy (QOL-DN) score from baseline at 18 months. Norfolk QOL-DN is a 35-item questionnaire sensitive to small-fiber, large-fiber and autonomic nerve function in which a higher score indicates worsening in QOL (range: -4 to 136) [34]. In the APOLLO study, prior TTR stabilizer use of either tafamidis or diflunisal (yes/no) was a stratification factor at randomization. Patients with prior tafamidis or diflunisal use were required to complete a 14-day washout period before the start of study drug administration in APOLLO; the reason for discontinuation, as entered and chosen by the investigator, was recorded.

\section{Post hoc analyses}

The data for the post hoc analyses come from above studies approved by central and local institutional review boards or ethics committees and conducted according to the Good Clinical Practice guidelines of the International Conference on Harmonization and the WHO Declaration of Helsinki. All the participants provided written informed consent.

Post hoc analyses of the Phase II OLE and APOLLO studies were conducted to explore the safety and PD of patisiran alone or with concomitant TTR stabilizer use, and safety and efficacy of patisiran in patients with prior TTR stabilizer use, respectively. Phase II OLE data were analyzed to determine safety (adverse events [AEs], serious AEs [SAEs]) and serum TTR reduction over 24 months, in patients who received patisiran therapy alone, and in those who received patisiran concomitantly with tafamidis or diflunisal. APOLLO data were analyzed to evaluate safety and changes in mNIS+7 and Norfolk QOL-DN at 18 months in patients receiving study drug who had no history of prior TTR stabilizer use and those who had received prior treatment with either tafamidis or diflunisal.

\section{Results}

\section{Phase II OLE experience: patisiran in patients with concomitant TTR stabilizer use}

A total of 27 patients enrolled in the Phase II OLE study; of these, seven patients (25.9\%) did not receive a TTR stabilizer during the Phase II OLE (patisiran alone group), 13 (48.1\%) received patisiran with concomitant tafamidis and seven $(25.9 \%)$ received patisiran with concomitant diflunisal. Baseline demographics of patients from each treatment group are shown in Table 1.11 patients (40.7\%) met the criteria for the cardiac subpopulation, one in the patisiran alone group and five each in the concomitant tafamidis and concomitant diflunisal groups.

Median exposure was 736 days (range: 735 to 737 ) for the cohort with patisiran alone, 736 days (range: 19 to 747 days) for concomitant tafamidis and 421 days (range: 139 to 736 days) for concomitant diflunisal. The frequencies of AEs, including severe AEs, SAEs and AEs leading to discontinuation are shown in Table 2. Most patients across the three treatment groups experienced AEs that were mild or moderate in intensity. SAEs were reported in two $(28.6 \%)$, four $(30.8 \%)$ and one $(14.3 \%)$ patient receiving patisiran alone, patisiran with concomitant tafamidis or concomitant diflunisal, respectively, as shown in Table 3.

Median (range) serum TTR per cent change from baseline averaged over 24 months was similar regardless of whether a patient received patisiran alone $(-88.4 \%$ [-91.1 to -65.0]) or with concomitant tafamidis $(-79.9 \%$ [-93.3 to -74.4$])$ or diflunisal (-84.1\% [-90.4 to -70.7]) (Figure 1).

\section{APOLLO experience: patisiran in patients with prior TTR stabilizer use}

APOLLO enrolled 225 patients, of whom 106 patients (47.1\%) did not receive prior TTR stabilizers, 74 (32.9\%) reported prior tafamidis use and $45(20.0 \%)$ reported prior diflunisal use. The majority of patients with prior tafamidis use $(\mathrm{n}=46,62.2 \%)$ and prior diflunisal use $(\mathrm{n}=40,88.9 \%)$ discontinued their therapy specifically to enrol in APOLLO (Figure 2). A total of 23 patients (31.1\%) reported to have discontinued tafamidis and one patient $(2.2 \%)$ to have discontinued diflunisal due to disease progression. Baseline demographics and disease characteristics of patients in APOLLO with no prior TTR stabilizer use, those with prior tafamidis use and those with prior diflunisal use, are shown in Table 4. A total of 126 patients $(56.0 \%)$ met the criteria for the cardiac subpopulation; the proportion of patients included in the cardiac subpopulation was similar across patisiran-treated 
Table 1. Baseline demographics and characteristics of patients receiving patisiran alone, patisiran with concomitant

tafamidis and patisiran with concomitant diflunisal in the Phase II open-label extension study.

\begin{tabular}{|c|c|c|c|}
\hline Variables & Patisiran alone $(n=7)$ & Patisiran and tafamidis $(n=13)$ & Patisiran and diflunisal $(n=7)$ \\
\hline Median age, years (range) & $55(40-75)$ & $45(29-77)$ & $69(63-75)$ \\
\hline Sex, male, n (\%) & $4(57.1)$ & $9(69.2)$ & $5(71.4)$ \\
\hline \multicolumn{4}{|l|}{ Region $^{\dagger}, \mathrm{n}(\%)$} \\
\hline North America & $0(0)$ & $0(0)$ & $1(14.3)$ \\
\hline Western Europe & $6(85.7)$ & $13(100.0)$ & $6(85.7)$ \\
\hline Rest of World & $1(14.3)$ & $0(0)$ & $0(0)$ \\
\hline Median years since hATTR amyloidosis diagnosis (range) & $2(1-4)$ & $3(2-8)$ & $2(1-3)$ \\
\hline Genotype V30M, n (\%) & $4(57.1)$ & $9(69.2)$ & $7(100.0)$ \\
\hline \multicolumn{4}{|l|}{ FAP stage, n (\%) } \\
\hline 1: Unimpaired ambulation & $6(85.7)$ & $11(84.6)$ & $7(100.0)$ \\
\hline 2: Assistance with ambulation required & $1(14.3)$ & $2(15.4)$ & $0(0)$ \\
\hline \multicolumn{4}{|l|}{ Polyneuropathy disability score, n (\%) } \\
\hline I: preserved walking, sensory disturbances & $2(28.6)$ & $7(53.8)$ & $6(85.7)$ \\
\hline II: impaired walking without need for a stick or crutch & $4(57.1)$ & $4(30.8)$ & $1(14.3)$ \\
\hline IIIA: walking with one stick or crutch & $1(14.3)$ & $1(7.7)$ & $0(0)$ \\
\hline IIIB: walking with two sticks or crutches & $0(0)$ & $1(7.7)$ & $0(0)$ \\
\hline IV: confined to wheelchair or bedridden & $0(0)$ & $0(0)$ & $0(0)$ \\
\hline \multicolumn{4}{|l|}{ New York Heart Association class, n (\%) } \\
\hline 1 & $6(85.7)$ & $7(53.8)$ & $6(100.0)$ \\
\hline II & $1(14.3)$ & $6(46.2)$ & $0(0)$ \\
\hline III & $0(0)$ & $0(0)$ & $0(0)$ \\
\hline IV & $0(0)$ & $0(0)$ & $0(0)$ \\
\hline Missing & 0 & 0 & 1 \\
\hline Cardiac subpopulation $\ddagger$, $(\%)$ & $1(14.3)$ & $5(38.5)$ & $5(71.4)$ \\
\hline
\end{tabular}

Table 2. Safety summary of patients receiving patisiran alone, patisiran with concomitant tafamidis and patisiran with concomitant diflunisal in the Phase II open-label extension study.

\begin{tabular}{|lll|}
\hline Event, $n(\%)$ & Patisiran alone $(n=7)$ & Patisiran and tafamidis $(n=13)$ \\
\hline Any AE & $6(85.7)$ & $13(100.0)$ \\
\hline Any severe AE & $2(28.6)$ & $2(15.4)$ \\
\hline Any SAE & $2(28.6)$ & $4(30.8)$ \\
\hline AE leading to discontinuation & $1(14.3)$ & $0(0)$ \\
\hline Death & $1(14.3)^{\dagger}$ & $0(0)$ \\
\hline $\begin{array}{l}\dagger \text { Causes of death were myocardial infarction and gastro-oesophageal cancer, respectively, and both were deemed not drug-related by investigators. } \\
\text { AE: Adverse event; SAE: Serious adverse event. }\end{array}$
\end{tabular}

subgroups $(62.9,59.6,58.1 \%)$ but more variable among placebo-treated subgroups $(52.8,33.3,57.1 \%)$ in patients with no prior TTR stabilizer, prior tafamidis and prior diflunisal use, respectively (Table 4).

Safety and tolerability are shown in Table 5 with the majority of AEs mild or moderate in severity. The most common AEs across all the subgroups (no prior TTR stabilizer, prior tafamidis and prior diflunisal use) were diarrhoea (47.2, 25.7, 33.3\%, respectively) and peripheral edema (33.0, 17.6, 28.9\%, respectively). Among patients treated with patisiran, the proportion who experienced infusion-related reactions was similar across subgroups (no prior TTR stabilizer, $20.0 \%$; prior tafamidis, $17.0 \%$; and prior diflunisal use, $19.4 \%$ respectively). There were no clinically relevant changes in laboratory values related to patisiran, including platelet counts and indicators of liver or kidney function (data not shown).

Across the three patient groups evaluated, the mean change in mNIS +7 from baseline to 18 months trended consistently, demonstrating a mean stabilization or improvement for all patisiran-treated groups and a deterioration for the placebo-treated groups (Figure 3A). The mean (standard deviation) $\mathrm{mNIS}+7$ change from baseline to 18 months for the patients receiving patisiran or placebo, by subgroup, was: -3.4 (21.0) and 24.9 points (20.5), respectively, for study drug alone; $-6.3(16.2)$ and 17.5 points (12.7), respectively, with prior tafamidis; and -2.8 (14.9) and 51.4 points (22.6), respectively, with prior diflunisal. A similar trend was seen for the mean change in 
Table 3. Summary of serious adverse events in the Phase II open-label extension study.

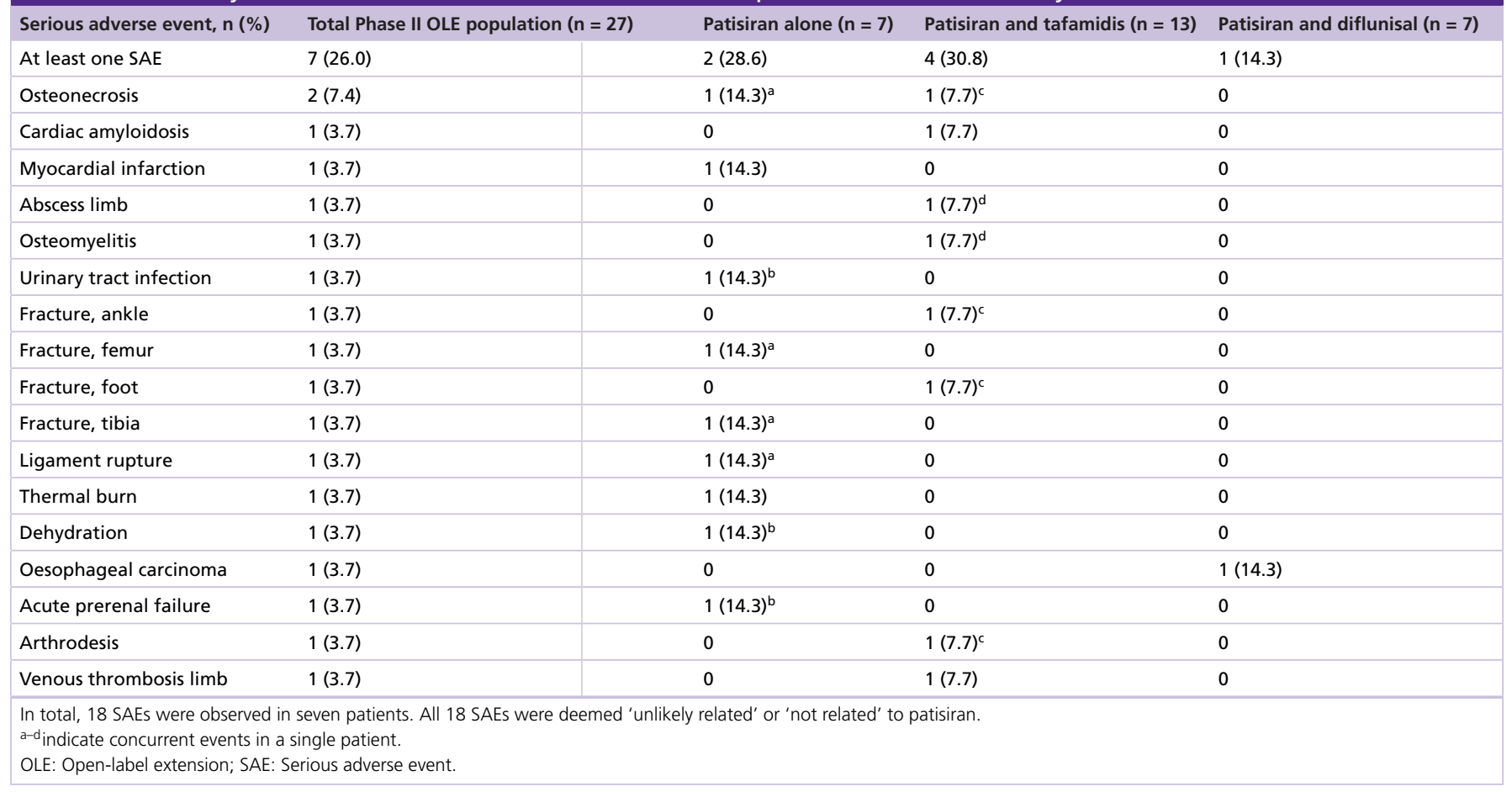

\section{Median TTR change (\%) from baseline averaged over 24 months, (range)}

Patisiran alone $(n=7)$

$-88.4(-91.1$ to -65.0$)$
Patisiran and tafamidis $(n=13)$

$-79.9(-93.3$ to -74.4$)$
Patisiran and diflunisal $(n=7)$

$-84.1(-90.4$ to -70.7$)$

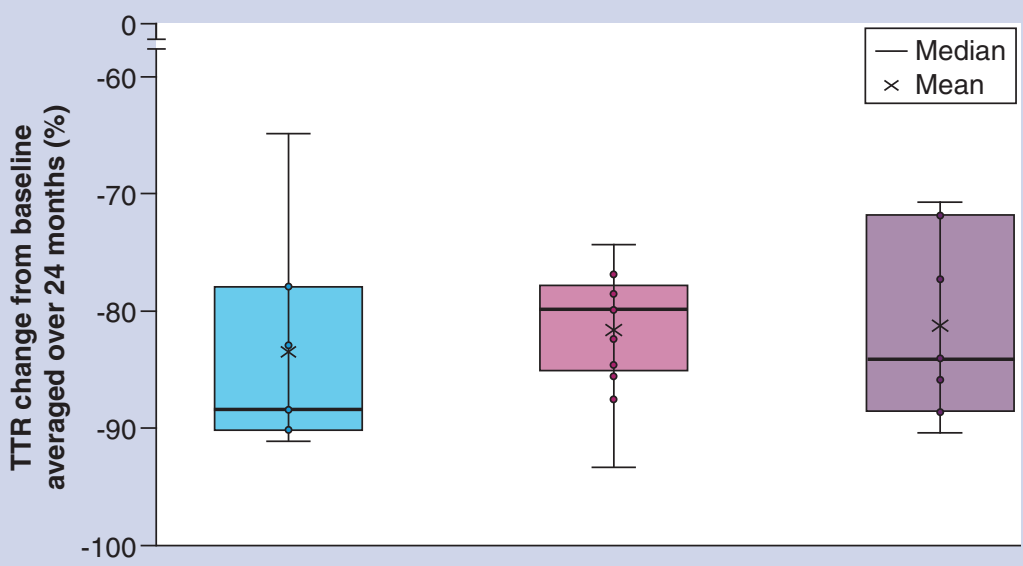

Figure 1. Serum transthyretin percent change from baseline averaged over 24 months among patients receiving patisiran alone, patisiran with concomitant tafamidis and patisiran with concomitant diflunisal in the Phase II open-label extension study. Median (range) serum TTR per cent change from baseline averaged over 24 months was similar regardless of whether a patient received patisiran alone $(-88.4 \%$ [-91.1 to -65.0$])$ or with concomitant tafamidis $(-79.9 \%$ [-93.3 to -74.4$])$ or diflunisal $(-84.1 \%$ [-90.4 to -70.7$])$. TTR: Transthyretin. 
Table 4. Baseline demographics and characteristics of patients with no prior transthyretin stabilizer use, those with prior tafamidis use and those with prior diflunisal use in the APOLLO study.

\begin{tabular}{|c|c|c|c|c|c|c|}
\hline \multirow[t]{2}{*}{ Variables } & \multicolumn{2}{|c|}{ No prior TTR stabilizer use } & \multicolumn{2}{|c|}{ Prior tafamidis use } & \multicolumn{2}{|c|}{ Prior diflunisal use } \\
\hline & Placebo $(n=36)$ & Patisiran $(n=70)$ & Placebo $(n=27)$ & Patisiran $(n=47)$ & Placebo $(n=14)$ & Patisiran $(n=31)$ \\
\hline Median age, years (range) & $62.5(36-80)$ & $61(24-77)$ & $63(34-77)$ & $64(27-83)$ & $66(46-75)$ & $62(35-75)$ \\
\hline Sex, male, n (\%) & $25(69.4)$ & $51(72.9)$ & $22(81.5)$ & $33(70.2)$ & $11(78.6)$ & $25(80.6)$ \\
\hline \multicolumn{7}{|l|}{ Region $^{\dagger}, \mathrm{n}(\%)$} \\
\hline North America & $5(13.9)$ & $16(22.9)$ & $0(0)$ & $0(0)$ & $5(35.7)$ & $21(67.7)$ \\
\hline Western Europe & $14(38.9)$ & $23(32.9)$ & $17(63.0)$ & $34(72.3)$ & $5(35.7)$ & $5(16.1)$ \\
\hline Rest of World & $17(47.2)$ & $31(44.3)$ & $10(37.0)$ & $13(27.7)$ & $4(28.6)$ & $5(16.1)$ \\
\hline $\begin{array}{l}\text { Median years since hATTR amyloidosis } \\
\text { diagnosis (range) }\end{array}$ & $0.7(0.1-16.5)$ & $1.1(0.0-21.0)$ & $2.1(0.0-7.7)$ & $1.9(0.2-17.5)$ & $2.9(0.4-13.0)$ & $1.9(0.0-11.9)$ \\
\hline \multicolumn{7}{|l|}{ TTR genotype, n (\%) } \\
\hline V30M & $17(47.2)$ & $25(35.7)$ & $18(66.7)$ & $22(46.8)$ & $5(35.7)$ & $9(29.0)$ \\
\hline $\begin{array}{l}\text { With onset of disease before } 50 \text { years } \\
\text { of age }\end{array}$ & $6(16.7)$ & $6(8.6)$ & $3(11.1)$ & $6(12.8)$ & $1(7.1)$ & $1(3.2)$ \\
\hline Non-V30M & $19(52.8)$ & $45(64.3)$ & $9(33.3)$ & $25(53.2)$ & 9 (64.3) & $22(71.0)$ \\
\hline \multicolumn{7}{|l|}{ FAP stage, $n(\%)$} \\
\hline 1: Unimpaired ambulation & $17(47.2)$ & $31(44.3)$ & $15(55.6)$ & $19(40.4)$ & $5(35.7)$ & $17(54.8)$ \\
\hline 2: Assistance with ambulation required & $18(50.0)$ & $39(55.7)$ & $12(44.4)$ & $28(59.6)$ & $9(64.3)$ & $14(45.2)$ \\
\hline 3: Wheelchair bound/bedridden & $1(2.8)$ & $0(0)$ & $0(0)$ & $0(0)$ & $0(0)$ & $0(0)$ \\
\hline \multicolumn{7}{|l|}{ Polyneuropathy disability score, n (\%) } \\
\hline I: preserved walking, sensory disturbances & $12(33.3)$ & $17(24.3)$ & $6(22.2)$ & $7(14.9)$ & $2(14.3)$ & $12(38.7)$ \\
\hline $\begin{array}{l}\text { II: impaired walking without need for a } \\
\text { stick or crutch }\end{array}$ & $6(16.7)$ & $20(28.6)$ & $11(40.7)$ & $17(36.2)$ & $6(42.9)$ & $6(19.4)$ \\
\hline IIIA: walking with one stick or crutch & $10(27.8)$ & $19(27.1)$ & $8(29.6)$ & $13(27.7)$ & $4(28.6)$ & $9(29.0)$ \\
\hline IIIB: walking with two sticks or crutches & $7(19.4)$ & $14(20.0)$ & $2(7.4)$ & $10(21.3)$ & $2(14.3)$ & $4(12.9)$ \\
\hline IV: confined to wheelchair or bedridden & $1(2.8)$ & $0(0)$ & $0(0)$ & $0(0)$ & $0(0)$ & $0(0)$ \\
\hline \multicolumn{7}{|l|}{ New York Heart Association class, n (\%) } \\
\hline 1 & $19(52.8)$ & $33(47.1)$ & $15(57.7)$ & $18(38.3)$ & $6(42.9)$ & $19(63.3)$ \\
\hline II & $17(47.2)$ & $37(52.9)$ & $11(42.3)$ & $29(61.7)$ & $8(57.1)$ & $11(36.7)$ \\
\hline III & $0(0)$ & $0(0)$ & $0(0)$ & $0(0)$ & $0(0)$ & $0(0)$ \\
\hline IV & $0(0)$ & $0(0)$ & $0(0)$ & $0(0)$ & $0(0)$ & $0(0)$ \\
\hline Missing & 0 & 0 & 1 & 0 & 0 & 1 \\
\hline Cardiac subpopulation ${ }^{\ddagger}, \mathrm{n}(\%)$ & $19(52.8)$ & $44(62.9)$ & $9(33.3)$ & $28(59.6)$ & $8(57.1)$ & $18(58.1)$ \\
\hline $\begin{array}{l}\text { Median months on prior TTR stabilizer, } \mathrm{n} \\
\text { (range) }\end{array}$ & $\mathrm{N} / \mathrm{A}$ & $\mathrm{N} / \mathrm{A}$ & $13.8(1.0-43.0)$ & $12.4(1.3-108.0)$ & $10.6(0.1-133.6)$ & $9.9(0.5-85.9)$ \\
\hline $\begin{array}{l}\text { Median days from discontinuation of TTR } \\
\text { stabilizer to start of study drug, } n \text { (range) }\end{array}$ & $\mathrm{N} / \mathrm{A}$ & $\mathrm{N} / \mathrm{A}$ & $22(15-148)$ & $30(15-318)$ & $13.5(6-113)$ & $21(4-1037)$ \\
\hline Median baseline mNIS $+7, \mathrm{n}$ (range) & $72.1(11-154)$ & $80.5(9-165)$ & $70.8(17-132)$ & $87.3(14-152)$ & $76.3(17-137)$ & $65.5(8-163)$ \\
\hline Median baseline Norfolk QOL-DN, n (range) & $50.0(14-111)$ & $67.5(5-119)$ & $54.0(17-91)$ & $62.0(10-113)$ & $61.0(8-83)$ & $49.0(7-95)$ \\
\hline \multicolumn{7}{|c|}{$\begin{array}{l}\text { †North America: USA, Canada; Western Europe: France, Germany, Italy, The Netherlands, Portugal, Spain, Sweden, and UK; Rest of World: Argentina, Brazil, Bulgaria, Cyprus, Japan, } \\
\text { Mexico, South Korea, Taiwan and Turkey. } \\
\text { ¥Prespecified cardiac subpopulation defined as left ventricular wall thickness } \geq 1.3 \mathrm{~cm} \text { and no medical history of aortic valve disease or hypertension. } \\
\text { FAP: Familial amyloidotic polyneuropathy; hATTR: Hereditary transthyretin-mediated; mNIS+7: Modified Neuropathy Impairment Score +7; Norfolk QOL-DN: Norfolk Quality of Life- } \\
\text { Diabetic Neuropathy; SD: Standard deviation; TTR: Transthyretin. }\end{array}$} \\
\hline
\end{tabular}

Table 5. Safety summary of patients with no prior transthyretin stabilizer use, those with prior tafamidis use and those with prior diflunisal use in the APOLLO study.

\begin{tabular}{|c|c|c|c|c|c|c|}
\hline \multirow[t]{2}{*}{ Event, n (\%) } & \multicolumn{2}{|c|}{ No prior TTR stabilizer use } & \multicolumn{2}{|c|}{ Prior tafamidis use } & \multicolumn{2}{|c|}{ Prior diflunisal use } \\
\hline & Placebo $(n=36)$ & Patisiran $(n=70)$ & Placebo $(n=27)$ & Patisiran $(n=47)$ & Placebo $(n=14)$ & Patisiran $(n=31)$ \\
\hline Any $A E$ & $35(97.2)$ & $68(97.1)$ & $26(96.3)$ & $45(95.7)$ & $14(100.0)$ & $30(96.8)$ \\
\hline Any severe $A E$ & $14(38.9)$ & $30(42.9)$ & $8(29.6)$ & $8(17.0)$ & $6(42.9)$ & 4 (12.9) \\
\hline Any SAE & $14(38.9)$ & $29(41.4)$ & $12(44.4)$ & $20(42.6)$ & $5(35.7)$ & $5(16.1)$ \\
\hline $\begin{array}{l}\text { AE leading to treatment } \\
\text { discontinuation }\end{array}$ & 5 (13.9) & $6(8.6)$ & $4(14.8)$ & $1(2.1)$ & $2(14.3)$ & $0(0)$ \\
\hline AE leading to study withdrawal & $5(13.9)$ & $6(8.6)$ & $3(11.1)$ & $1(2.1)$ & $1(7.1)$ & $0(0)$ \\
\hline Death & $4(11.1)^{\dagger}$ & $5(7.1)^{\dagger}$ & $2(7.4)^{\dagger}$ & $2(4.3)^{\dagger}$ & $0(0)$ & $0(0)$ \\
\hline
\end{tabular}

$\dagger$ Deemed not drug-related by investigators.

AE: Adverse event; SAE: Serious adverse event; TTR: Transthyretin. 


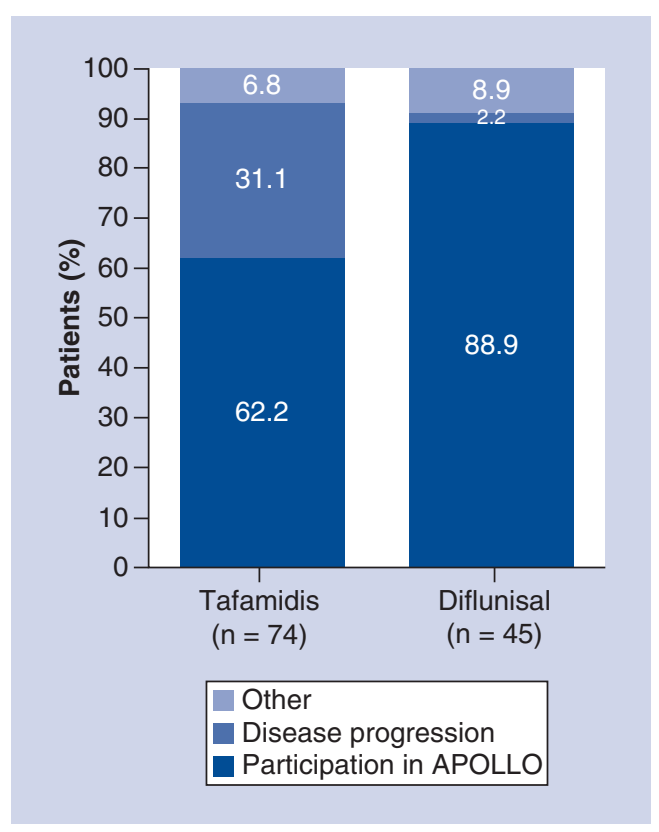

Figure 2. Reasons for discontinuation of transthyretin stabilizers prior to enrolment in the APOLLO study. The majority of patients with prior tafamidis use $(n=46,62.2 \%)$ and prior diflunisal use $(n=40,88.9 \%)$ discontinued their therapy specifically to enrol in APOLLO.

Norfolk QOL-DN from baseline to 18 months (Figure 3B). The mean (standard deviation) Norfolk QOL-DN change from baseline to 18 months for the patients receiving patisiran or placebo, by subgroup, was: -6.5 (24.3) and 19.7 points (23.7), respectively, for study drug alone; -0.9 (17.6) and 17.9 points (20.2), respectively, with prior tafamidis; and 2.1 (19.1) and 25.2 points (15.5), respectively, with prior diflunisal.

\section{Discussion}

With the recent approvals of new therapies for hATTR amyloidosis [14,15,17,19-27], clinicians are making decisions about which therapy, or therapies, to use to treat this rapidly progressive disease in an evolving treatment landscape. Clinical manifestations of the disease vary widely; phenotype can predominantly present with neuropathic or cardiac manifestations, but a majority of patients have a mixed phenotype with both polyneuropathy and cardiomyopathy. The treatment decision process is further complicated by the varying levels of clinical evidence across different populations. Thus, there is a growing interest to understand the role of each of these therapies in managing patients with different manifestations of this disease.

Here, we present data from the clinical development programme of patisiran in patients with hATTR amyloidosis with polyneuropathy to help inform decisions about the use of patisiran with a concomitant TTR stabilizer treatment or following prior use of a TTR stabilizer. 27 patients were enrolled in the Phase II OLE with approximately $40 \%$ of patients meeting the prespecified criteria for the cardiac subpopulation, which demonstrates a significant representation of patients with mixed phenotype disease in this study. Data from this small cohort of patients who received patisiran with concomitant tafamidis or diflunisal suggest that the safety profiles in these patients were consistent with the safety profiles of the respective therapies as monotherapy $[7,11,13]$. Serum TTR reduction was similar regardless of whether a patient received patisiran alone or with concomitant tafamidis or diflunisal, suggesting that the PD effect of patisiran is unaffected by concomitant TTR stabilizer use.

We also explored the safety and potential efficacy of patisiran in patients who had received prior TTR stabilizer therapy, using data from the randomized, placebo-controlled patisiran Phase III APOLLO study. Approximately a third of patients enrolled in APOLLO were previously treated with tafamidis and a fifth of patients were previously treated with diflunisal. Many patients with prior TTR stabilizer use were reported to have discontinued their TTR stabilizer due to disease progression, with the majority of the other patients discontinuing specifically to participate in the APOLLO study. Approximately half of the patients in this post hoc analysis of the APOLLO study also met the criteria for the cardiac subpopulation, which also underscores the high proportion of patients with mixed phenotype disease represented in this study.

The analyses presented here showed similar safety profiles in patients with prior TTR stabilizer use and those with no history of TTR stabilizer therapy. Furthermore, the safety profiles in the three patient groups were consistent 
(A)

\begin{tabular}{|c|c|c|c|c|c|c|}
\hline & \multicolumn{2}{|c|}{ No prior stabilizer } & \multicolumn{2}{c|}{ Prior tafamidis } & \multicolumn{2}{c|}{ Prior diflunisal } \\
\hline & Placebo & Patisiran & Placebo & Patisiran & Placebo & Patisiran \\
\hline $\mathrm{n}$ & 26 & 62 & 19 & 45 & 13 & 31 \\
\hline
\end{tabular}

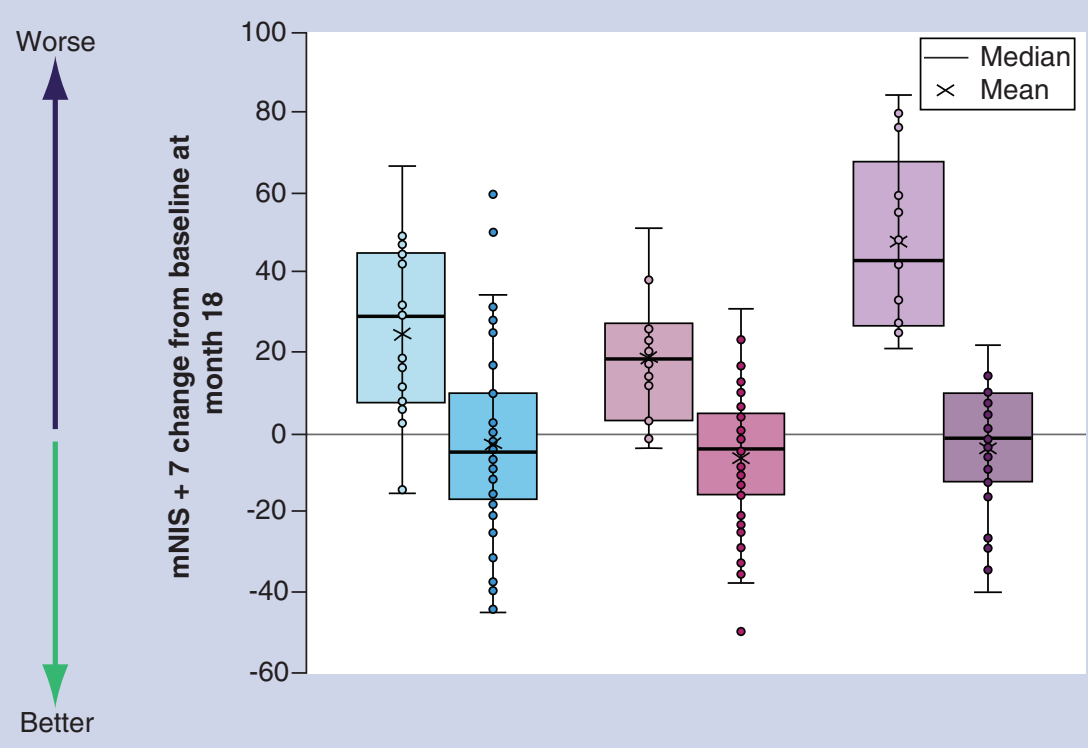

(B)

\begin{tabular}{|c|c|c|c|c|c|c|}
\hline & \multicolumn{2}{|c|}{ No prior stabilizer } & \multicolumn{2}{|c|}{ Prior tafamidis } & \multicolumn{2}{c|}{ Prior diflunisal } \\
\hline Placebo & Patisiran & Placebo & Patisiran & Placebo & Patisiran \\
\hline $\mathrm{n}$ & 25 & 61 & 18 & 45 & 12 & 31 \\
\hline
\end{tabular}

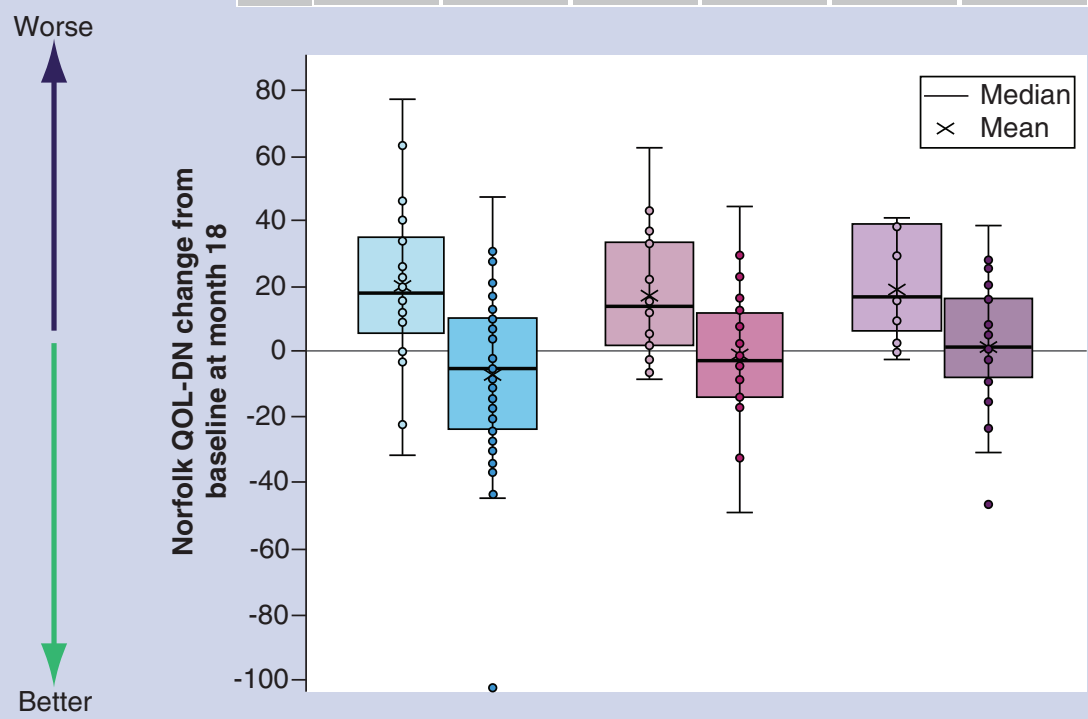

Figure 3. Change from baseline to 18 months in APOLLO patients according to prior transthyretin stabilizer use for (A) mNIS+7 and (B) Norfolk QOL-DN. Across the groups, the mean change in mNIS +7 from baseline to 18 months trended consistently, demonstrating a mean stabilization or improvement for all patisiran-treated groups and a deterioration for the placebo-treated groups. A similar trend was seen for the mean change in Norfolk QOL-DN from baseline to 18 months.

mNIS+7: Modified Neuropathy Impairment Score +7; QOL-DN: Quality of Life-Diabetic Neuropathy. 
with that observed in the overall APOLLO population, with diarrhoea and peripheral oedema being the most common AEs and low rates of AEs leading to treatment discontinuation [7]. Thus, the totality of the APOLLO experience highlights that patisiran is well tolerated, with an acceptable benefit:risk profile, whether used in patients with a history of prior TTR stabilizer use or in those who are TTR stabilizer naive.

Analyses of the APOLLO data show that patients who had prior tafamidis or diflunisal use had a similar stabilization or improvement in polyneuropathy symptoms from baseline to 18 months, as measured by mNIS+7, as those who had no history of prior TTR stabilizer use. A similar trend was also seen in patient QOL, as measured by the Norfolk QOL-DN questionnaire. Overall, these data from APOLLO indicate that the safety and efficacy of patisiran appear to be unaffected by prior TTR stabilizer use. Therefore, patients who discontinue a TTR stabilizer treatment due to disease progression, or for other reasons, may stabilize or improve their polyneuropathy and QOL by switching to patisiran.

However, patisiran treatment may not be able to fully reverse deterioration in neurological function and QOL that occurs during TTR stabilizer treatment. Previous studies have reported worsening of polyneuropathy and QOL on average after treatment with tafamidis $[13,35,36]$, as well as polyneuropathy progression in those treated with diflunisal [11]. Evidence regarding the ability of patisiran to impact polyneuropathy progression is available from the ongoing Global OLE study (NCT02510261). After 12 months of patisiran treatment in the ongoing Global OLE, patisiran halted polyneuropathy progression (measured by $\mathrm{mNIS}+7$ score) on average in patients who had previously received placebo in APOLLO. However, after 12 months of patisiran treatment in the Global OLE, these patients' neuropathy impairment had not returned to their APOLLO baseline value following neurological deterioration experienced while receiving placebo for 18 months in APOLLO [37]. Similar trends were observed for QOL [37]. These data highlight the urgency to initiate patisiran as early in the disease course as possible to prevent further disability and morbidity.

In the absence of direct comparisons, a previous analysis evaluated patisiran and tafamidis via an indirect treatment comparison to understand the comparative efficacy of each individual monotherapy to prevent disease progression in patients with hATTR amyloidosis with polyneuropathy [38]. The analysis of these treatments as monotherapy suggests that patisiran has a greater treatment effect than tafamidis across measures of neurological impairment and QOL in patients with hATTR amyloidosis with polyneuropathy.

It is important to note that the data presented here are limited to the experience seen in patients with hATTR amyloidosis with polyneuropathy and specific to polyneuropathy endpoints; thus, additional data and studies are needed in patients with ATTR amyloidosis with significant cardiomyopathy. Additional data can be expected as part of the ongoing Phase III APOLLO-B clinical study (NCT03997383), which is enrolling patients with ATTR amyloidosis with cardiomyopathy and allows enrolment of patients receiving concomitant tafamidis [39]. Other limitations of the investigation described here include the post hoc nature of the analyses; additional prospective studies are needed to further elucidate the roles of different therapies in the treatment of ATTR amyloidosis.

\section{Conclusion}

In conclusion, these analyses suggest that the safety and PD profile of patisiran are not impacted by concomitant TTR stabilizer use. Additionally, these data indicate that patients benefit from patisiran treatment regardless of concomitant or prior use of TTR stabilizers.

\section{Author contributions}

All authors were responsible for the conception and design of the work, and also analyzed and interpreted the data. All authors revised the manuscript critically for important intellectual content, approved the final version to be published, and agreed to be accountable for all aspects of the work in ensuring that questions related to the accuracy or integrity of any part of the work are appropriately investigated and resolved.

\section{Acknowledgments}

The authors want to thank patients, their families, investigators, study staff and collaborators for their participation in the Phase II OLE and APOLLO studies.

\section{Financial \& competing interests disclosure}

This study was funded by Alnylam Pharmaceuticals Inc. All authors are employees of Alnylam Pharmaceuticals Inc. All authors are shareholders and no authors hold patents in Alnylam Pharmaceuticals Inc. Open access was funded by Alnylam Pharmaceuticals 
Inc. The authors have no other relevant affiliations or financial involvement with any organization or entity with a financial interest in or financial conflict with the subject matter or materials discussed in the manuscript apart from those disclosed.

Editorial support was provided by Anastasia McManus, PharmD, RPh, of Alnylam Pharmaceuticals Inc., USA, Katherine Alfond, PharmD, RPh, postdoctoral fellow of Alnylam Pharmaceuticals Inc., USA, and Colette Szarka, of Adelphi Communications Ltd, Macclesfield, UK, in accordance with the Good Publication Practice (GPP3) guidelines, funded by Alnylam Pharmaceuticals Inc., USA.

\section{Ethical conduct of research}

The data for the post hoc analyses come from studies approved by central and local institutional review boards or ethics committees and conducted according to the Good Clinical Practice guidelines of the International Conference on Harmonization and the WHO Declaration of Helsinki. All the participants provided written informed consent.

\section{Data sharing statement}

The authors certify that this manuscript reports the secondary analysis of clinical trial data that are owned by Alnylam Pharmaceuticals, and that the use of these data is in accordance with the terms (if any) agreed upon their receipt.

Open access

This work is licensed under the Attribution-NonCommercial-NoDerivatives 4.0 Unported License. To view a copy of this license, visit http://creativecommons.org/licenses/by-nc-nd/4.0/

\section{References}

Papers of special note have been highlighted as: $\bullet$ of interest

1. Hanna M. Novel drugs targeting transthyretin amyloidosis. Curr. Heart Fail. Rep. 11(1), 50-57 (2014).

2. Hawkins PN, Ando Y, Dispenzeri A, Gonzalez-Duarte A, Adams D, Suhr OB. Evolving landscape in the management of transthyretin amyloidosis. Ann. Med. 47(8), 625-638 (2015).

- A comprehensive review of diagnosis, disease characteristics, natural history and therapeutic strategies in transthyretin-mediated amyloidosis.

3. Castaño A, Drachman BM, Judge D, Maurer MS. Natural history and therapy of TTR-cardiac amyloidosis: emerging disease-modifying therapies from organ transplantation to stabilizer and silencer drugs. Heart Fail. Rev. 20(2), 163-178 (2015).

4. Gertz MA, Kyle RA, Thibodeau SN. Familial amyloidosis: a study of 52 North American-born patients examined during a 30-year period. Mayo Clin. Proc. 67(5), 428-440 (1992).

5. Sattianayagam PT, Hahn AF, Whelan CJ et al. Cardiac phenotype and clinical outcome of familial amyloid polyneuropathy associated with transthyretin alanine 60 variant. Eur. Heart J. 33(9), 1120-1127 (2012).

6. Swiecicki PL, Zhen DB, Mauermann ML et al. Hereditary ATTR amyloidosis: a single-institution experience with 266 patients. Amyloid 22(2), 123-131 (2015).

7. Adams D, Gonzalez-Duarte A, O'Riordan WD et al. Patisiran, an RNAi therapeutic, for hereditary transthyretin amyloidosis. N. Engl. J. Med. 379(1), 11-21 (2018).

- Pivotal Phase III study describing the efficacy and safety of patisiran in patients with hereditary transthyretin-mediated (hATTR) amyloidosis with polyneuropathy, which acts as a data source for this secondary analysis.

8. Benson MD, Waddington-Cruz M, Berk JL et al. Inotersen treatment for patients with hereditary transthyretin amyloidosis. N. Engl. J. Med. 379(1), 22-31 (2018).

9. Coelho T, Maurer MS, Suhr OB. THAOS - The Transthyretin Amyloidosis Outcomes Survey: initial report on clinical manifestations in patients with hereditary and wild-type transthyretin amyloidosis. Curr. Med. Res. Opin. 29(1), 63-76 (2013).

10. Rapezzi C, Quarta CC, Obici L et al. Disease profile and differential diagnosis of hereditary transthyretin-related amyloidosis with exclusively cardiac phenotype: an Italian perspective. Eur. Heart J. 34(7), 520-528 (2013).

11. Berk JL, Suhr OB, Obici L et al. Repurposing diflunisal for familial amyloid polyneuropathy: a randomized clinical trial. JAMA 310(24), 2658-2667 (2013).

- Phase II/III clinical trial to determine the effect of diflunisal on polyneuropathy progression in patients with hATTR amyloidosis.

12. Coelho T, Maia LF, da Silva AM et al. Long-term effects of tafamidis for the treatment of transthyretin familial amyloid polyneuropathy. J. Neurol. 260(11), 2802-2814 (2013).

13. Coelho T, Maia LF, Martins da Silva A et al. Tafamidis for transthyretin familial amyloid polyneuropathy: a randomized, controlled trial. Neurology 79(8), 785-792 (2012). 
- Phase II/III study to evaluate the efficacy and safety of tafamidis in patients with early-stage hATTR amyloidosis with polyneuropathy.

14. Pfizer. VYNDAQEL ${ }^{\circledR}$ (tafamidis) receives approval in Brazil by ANVISA for the treatment of transthyretin familial amyloid polyneuropathy. 2016. www.pfizer.com/sites/default/files/news/Brazil\%20Approval\%20Press\%20Statement\%2011-7-16_0.pdf

15. European Medicines Agency. Summary of product characteristics: Vyndaqel. Accessed 27th May 2020. www.ema.europa.eu/en/documents/product-information/vyndaqel-epar-product-information_en.pdf

16. Waddington Cruz M, Benson MD. A review of tafamidis for the treatment of transthyretin-related amyloidosis. Neurol. Ther. 4(2), 61-79 (2015).

17. Pharmaceuticals and Medical Devices Agency. Report on the deliberation results: Vyndaqel capsules $20 \mathrm{mg}$. (2013). www.pmda.go.jp/files/000153750.pdf

18. Maurer MS, Schwartz JH, Gundapaneni B et al. Tafamidis treatment for patients with transthyretin amyloid cardiomyopathy. N. Engl. J. Med. 379(11), 1007-1016 (2018).

19. Pfizer. US prescribing information: VYNDAQEL ${ }^{\circledR}$ (tafamidis meglumine) capsules, for oral administration and VYNDAMAX ${ }^{T M}$ (tafamidis) capsules, for oral administration. (2019). www.fda.gov/media/126283/download

20. Akcea Therapeutics. US prescribing information: TEGSEDI (inotersen) injection, for subcutaneous use - October 2019 update. (2019). https://tegsedi.com/prescribing-information.pdf

21. Alnylam Pharmaceuticals. Alnylam announces approval in Japan of ONPATTRO ${ }^{\circledR}$ for the treatment of hereditary ATTR amyloidosis with polyneuropathy. 2019. https://investors.alnylam.com/press-release?id=23886

22. Alnylam Pharmaceuticals Inc. US prescribing information: ONPATTRO (patisiran) lipid complex injection, for intravenous use. (2020). www.accessdata.fda.gov/drugsatfda_docs/label/2020/210922s007lbl.pdf

23. CADTH. Patisiran. (2019). www.cadth.ca/patisiran

24. European Medicines Agency. Summary of product characteristics: Tegsedi $284 \mathrm{mg}$ solution for injection in pre-filled syringe. Accessed 27th May 2020. www.ema.europa.eu/documents/product-information/tegsedi-epar-product-information_en.pdf

25. European Medicines Agency. Summary of product characteristics: Onpattro $2 \mathrm{mg} / \mathrm{mL}$ concentrate for solution for infusion. Accessed 27th May 2020. www.ema.europa.eu/documents/product-information/onpattro-epar-product-information_en.pdf

26. swissmedic. Abbreviated information for health care professionals for ONPATTRO $10 \mathrm{mg} / 5 \mathrm{ml}$, concentrate for solution for infusion (version September 2019). (2019). www.swissmedicinfo.ch

27. Alnylam Pharmaceuticals. Alnylam announces approval in Brazil of ONPATTRO ${ }^{\circledR}$ for the treatment of hereditary ATTR amyloidosis with polyneuropathy. (2020). https://investors.alnylam.com/press-release?id=24606

28. Coelho T, Adams D, Silva A et al. Safety and efficacy of RNAi therapy for transthyretin amyloidosis. N. Engl. J. Med. 369(9), 819-829 (2013).

29. Dubrey S, Ackermann E, Gillmore J. The transthyretin amyloidoses: advances in therapy. Postgrad. Med. J. 91(1078), 439-448 (2015).

30. Suhr OB, Coelho T, Buades J et al. Efficacy and safety of patisiran for familial amyloidotic polyneuropathy: a phase II multi-dose study. Orphanet J. Rare Dis. 10, 109 (2015).

31. Solomon SD, Adams D, Kristen A et al. Effects of patisiran, an RNA interference therapeutic, on cardiac parameters in patients with hereditary transthyretin-mediated amyloidosis. Circulation 139(4), 431-443 (2019).

- Describes the prespecified subpopulation of patients with evidence of cardiac amyloid involvement at baseline in the pivotal Phase III APOLLO study of patisiran in patients with hATTR amyloidosis with polyneuropathy.

32. Berk J, Adams D, Suhr O et al. Long-term, open-label clinical experience with patisiran, an investigational RNAi therapeutic for patients with hereditary transthyretin-mediated (hATTR) amyloidosis with polyneuropathy. Orphanet J. Rare Dis. 12(Suppl. 1), S165, Abstract P45 (2017).

- A Phase II open-label extension study investigating the long-term safety and efficacy of patisiran in patients with hATTR amyloidosis with polyneuropathy, which acts as a data source for this secondary analysis.

33. Suanprasert N, Berk JL, Benson MD et al. Retrospective study of a TTR FAP cohort to modify NIS+7 for therapeutic trials. J. Neurol. Sci. 344(1-2), 121-128 (2014).

34. Vinik EJ, Vinik AI, Paulson JF et al. Norfolk QOL-DN: validation of a patient reported outcome measure in transthyretin familial amyloid polyneuropathy. J. Peripher. Nerv. Syst. 19(2), 104-114 (2014).

35. Lozeron $\mathrm{P}$, Theaudin M, Mincheva $\mathrm{Z}$ et al. Effect on disability and safety of tafamidis in late onset of Met30 transthyretin familial amyloid polyneuropathy. Eur. J. Neurol. 20(12), 1539-1545 (2013).

36. Plante-Bordeneuve V, Gorram F, Salhi $\mathrm{H}$ et al. Long-term treatment of transthyretin familial amyloid polyneuropathy with tafamidis: a clinical and neurophysiological study. J. Neurol. 264(2), 268-276 (2017).

37. Polydefkis M, González Duarte A, Coelho T et al. Long-term safety and efficacy of patisiran in patients with hATTR amyloidosis: global OLE study. Peripheral Nerve Society (PNS), FL, USA (2019). 
38. Planté-Bordeneuve V, Lin H, Gollob J et al. An indirect treatment comparison of the efficacy of patisiran and tafamidis for the treatment of hereditary transthyretin-mediated amyloidosis with polyneuropathy. Expert Opin. Pharmacother. 20(4), 473-481 (2019).

- This indirect treatment comparison evaluates the efficacy of tafamidis and patisiran in patients with hATTR amyloidosis with polyneuropathy.

39. Alnylam Pharmaceuticals. Clinical trial: APOLLO-B: a study to evaluate patisiran in participants with transthyretin amyloidosis with cardiomyopathy (ATTR amyloidosis with cardiomyopathy). Report number: NCT03997383. Accessed 27th May 2020. https://clinicaltrials.gov/ct2/show/NCT03997383?term=NCT03997383\&draw=2\&rank=1 